\title{
EVALUATION OF THE SILVER SPECIES NATURE IN Ag-ITQ2 ZEOLITES BY THE CO OXIDATION REACTION
}

\author{
I. López-Hernández ${ }^{1}$, C. García ${ }^{2}$, V. Truttmann², S. Pollitt ${ }^{2}$, N. Barrabés ${ }^{2}$, G. Rupprechter ${ }^{2}$, F. Rey ${ }^{1}$, A. E. \\ Palomares ${ }^{1}$ \\ ${ }^{I}$ Instituto de Tecnología Química, Universitat Politècnica de València - Consejo Superior de Investigaciones \\ Científicas (UPV-CSIC), Valencia, España \\ ${ }^{2}$ Institute of Materials Chemistry, Technical University Vienna, Viena, Austria \\ Corresponding author: apalomar@iqn.upv.es
}

Keywords: CO oxidation, silver nanoclusters, silver species, silver zeolites, test reaction

\begin{abstract}
Silver containing zeolites are widely used in heterogeneous catalysis and their catalytic performance is strongly dependent on the nature of the metal species supported on the zeolites. In this work, Ag-ITQ2 zeolites have been prepared by different methods: incipient wetness impregnation, ion exchange and preformed Ag-nanoclusters supported on the zeolite. The samples have been pretreated with oxygen or hydrogen previously to the catalytic test in the $\mathrm{CO}$ oxidation reaction. The results allow to correlate the nature of the Ag-species with its catalytic activity in this reaction as metallic silver is active for the $\mathrm{CO}$ oxidation but cationic silver does not show significant activity. It is shown that $\mathrm{Ag}^{0}$ is formed on the catalysts prepared by impregnation even without a reduction treatment. On the contrary, Agnanoclusters form stable partially charged silver species that only can be reduced to $\mathrm{Ag}^{0}$ nanoparticles under severe thermal conditions. Silver species formed in the catalysts prepared by ion exchange also present a partial positive charge. We concluded that this reaction is a useful test for monitoring the nature and evolution of silver species in the zeolite and to determine the stability of the silver nanoclusters.
\end{abstract}




\section{1.- INTRODUCTION}

Silver-based catalysts are used in a wide range of applications such as the selective catalytic reduction of $\mathrm{NO}_{\mathrm{x}}$ [1], the selective catalytic oxidation of ammonia [2], the oxidation of styrene [3], the $\mathrm{CO}$ oxidation or even as antibacterial agents [4]. Among them, silver nanoparticles are a subject of actual interest as, at the nanoscale, enhanced reactivity and selectivity of these materials has been reported [5-7]. Further going down, under 100 atoms, metal nanoclusters, with unique geometric structure and electronic configuration represent an emerging field of research in heterogeneous catalysis [8-12]. The design of active sites at atomic size makes possible the metal nanostructure modification at molecular level [13]. In this way, monolayer protected clusters are composed by a metallic core structure stabilized with oligomers (called staples) holding metal atoms with partially charge state [14-15]. Therefore, they are an optimal type of model particles to study size and charge effect on the catalytic behavior of silver-based materials.

The catalytic activity of these materials depends strongly on the nature of silver species, which is determined mainly by the preparation methods, by the pretreatments, or by the reactions conditions [16-18]. These species can be ionic silver or clusters, in different size scale, holding metallic, cationic or partially charged state [16, 19-20]. In order to develop optimal silver based heterogeneous catalysts, detailed characterization of the different species is required. However, at this size and with low metal content, this becomes challenging, even with advanced spectroscopic techniques, due to the analyzer sensibility limits and to the possible sample damage [21]. For that reason, complementary studies to evaluate nature and changes in the metal sites are required. This can be done using some catalytic model reactions that are used as a tool or as a reference to identify these species and their evolution. The CO oxidation reaction can be used for this purpose as it is catalyzed by different metals as gold or silver supported on oxides [22-24].

In this paper we used the catalytic oxidation of $\mathrm{CO}$ as a tool to evaluate the nature and stability of the silver species present in some Ag-ITQ2 zeolites. The silver has been incorporated into the zeolite by different techniques such as incipient wetness impregnation, ion exchange and as silver-nanoclusters. The catalytic tests coupled with surface and structural characterization techniques discloses differences in the oxidation state of silver particles depending on the preparation protocols and in the catalyst treatment. 


\section{2.- EXPERIMENTAL}

\subsection{Catalyst Preparation}

The ITQ-2 zeolite with a Si/Al ratio of 10 was synthesized in our laboratory according to the literature [25]. Silver was incorporated to zeolites by three different methods: i) incipient wetness impregnation, ii) ion exchange, and iii) as silver-nanoclusters. The silver content was 1 wt. $\%$ in all cases.

The catalyst prepared by incipient wetness impregnation method was made by adding dropwise $3.5 \mathrm{~mL}$ of a silver nitrate $(0.0265 \mathrm{M})$ water solution into a $1 \mathrm{~g}$ of ITQ-2 zeolite. After impregnation, the samples were dried at $100^{\circ} \mathrm{C}$ during $24 \mathrm{~h}$, and then calcined at $250^{\circ} \mathrm{C}$ for $1 \mathrm{~h}$ and at $450^{\circ} \mathrm{C}$ for $2 \mathrm{~h}$.

The ion exchange was made with a solution of $\mathrm{AgNO}_{3}\left(9.28 \cdot 10^{-4} \mathrm{M}\right)$ while stirring during 24 hours at room temperature and in full darkness. The solid/solution ratio was $1 \mathrm{~g} / 100 \mathrm{~mL}$. The solid was recovered by filtration, washed with distilled water and dried overnight at $100^{\circ} \mathrm{C}$. The ion exchanged ITQ-2 zeolite was calcined at $250^{\circ} \mathrm{C}$ for $1 \mathrm{~h}$ and later on at $450^{\circ} \mathrm{C}$ for $2 \mathrm{~h}$.

The silver nanoclusters were synthesized based on the reported synthesis of $\mathrm{Ag}_{25}\left(\mathrm{SPhMe}_{3}\right)_{18} \mathrm{PPh}_{4}$ nanoclusters [26]. The purity of the synthesized $\mathrm{Ag}_{25}\left(\mathrm{SPhMe}_{3}\right)_{18} \mathrm{PPh}_{4}$ cluster solution was confirmed by UV-Vis, FTIR and TEM (Figures S1 and S2). The solution was dried and the amount of clusters necessary to obtain a $1 \mathrm{wt} . \%$ of silver on the zeolite, was redissolved in toluene and stirred with the ITQ-2 zeolite for $24 \mathrm{~h}$. The catalyst was separated from the solvent by centrifugation and decantation. The sample was dried at $80^{\circ} \mathrm{C}$ for $1 \mathrm{~h}$. In the impregnation, some aggregation of the initial $\mathrm{Ag}_{25}$ nanocluster occurred [26] for that reason these samples are named as $\mathrm{Ag}_{\mathrm{x}}$ nanoclusters.

\subsection{Catalytic tests}

The samples were pretreated under $\mathrm{O}_{2}\left(150^{\circ} \mathrm{C}, 1 \mathrm{~h}\right)$ or $\mathrm{H}_{2}\left(150^{\circ}\right.$ or $\left.400^{\circ} \mathrm{C}, 2 \mathrm{~h}\right)$ at atmospheric pressure before the reaction tests. The $\mathrm{CO}$ oxidation was carried out in a fixed-bed quartz reactor at atmospheric pressure. The reactor was loaded with $250 \mathrm{mg}$ of sieved catalyst (0.4$0.6 \mathrm{~mm}$ ) and the reaction was made with a flow rate gas of $500 \mathrm{~mL} \cdot \mathrm{min}^{-1}$ which led to a gas hour space velocity (GHSV) of $120000 \mathrm{~mL} \cdot \mathrm{g}^{-1} \cdot \mathrm{h}^{-1}$. The inlet gas concentration was $0.5 \% \mathrm{CO}$, 
$4 \% \mathrm{O}_{2}$ and $\mathrm{N}_{2}$ as balance gas. During the experiments, the temperature was increased in steps of $50^{\circ} \mathrm{C}$ from $100^{\circ} \mathrm{C}$ to $400-600^{\circ} \mathrm{C}$. The $\mathrm{CO}_{2}$ concentration was continuously registered by an infrared spectroscopy analyzer of Servomex Model 4900.

\subsection{Characterization}

UV-Vis DRS measurements were performed on a Varian Cary 5000 spectrophotometer equipped with a diffuse reflectance attachment, and the spectra were recorded at room temperature in the wavelength range from 200 to $800 \mathrm{~nm}$.

X-ray powder diffraction (XRD) was carried out using a Philips X'Pert (Cubix-Pro)-advance diffractometer operating at $45 \mathrm{kV}$ and $40 \mathrm{~mA}$, and coupled to a copper anode X-ray tube $(\mathrm{Cu}$ K $\alpha$ radiation, $\lambda=1.542 \AA$ ).

BET surface areas were determined from the nitrogen adsorption-desorption curves by the conventional multipoint technique with a Micromeritics ASAP 2420. The samples were pretreated at $400^{\circ} \mathrm{C}$ for 12 hours at high vacuum.

X-ray absorption fine structure (XAFS) measurements at the Ag K-edge (25.514 keV) were carried out at the BL22-CLAESS beamline at the ALBA synchrotron (Barcelona, Spain). Fresh samples were prepared as $5 \mathrm{~mm}$ pellets and mounted on the in situ reaction cell. Initially $\mathrm{Ar}$ was flowed through the Ag-ITQ2 and later a mixture of $\mathrm{O}_{2} / \mathrm{Ar}$ or $\mathrm{H}_{2} / \mathrm{Ar}$ was introduced. Heating process was done with a ramp of $5^{\circ} \mathrm{C} / \mathrm{min}$. After it, the sample was cooled down under inert atmosphere before $\mathrm{CO}$ oxidation. The reaction was done in a temperature programmed mode, with two steps at $150^{\circ} \mathrm{C}$ and $400^{\circ} \mathrm{C}$ for $30 \mathrm{~min}$. The data analysis was performed according to standard procedures using Ifeffit software.

\section{3.- RESULTS AND DISCUSSION}

Ag-ITQ2 catalysts prepared by different methods and with different pretreatments have been used to evaluate the nature of the silver species present in the zeolite by the $\mathrm{CO}$ oxidation reaction. The XRD pattern of the support (Figure S3) shows the characteristic peaks of the ITQ2 zeolite [25]. Same patterns, with minor modifications, are observed after the incorporation of the silver by the different protocols and no peaks related to silver species appear, excluding the formation of large silver particles (Figure S3). The materials have a 
high BET surface area around 450-500 $\mathrm{m}^{2} / \mathrm{g}$ with an external surface area of $350-450 \mathrm{~m}^{2} / \mathrm{g}$. The high surface area of the catalysts is due to the layered characteristics of the ITQ2 delaminated zeolite.

\subsection{Pretreatment effect}

The influence of the pretreatment applied to the Ag-zeolites on the formation of different silver species was studied with the samples prepared by wetness impregnation $\left(\mathrm{Ag}_{\text {imp }} / \mathrm{ITQ} 2\right)$ by their catalytic performance at the $\mathrm{CO}$ oxidation reaction. Three different pretreatments were used: oxidation and sample exposed to light ( $\mathrm{pretO}_{2 \_ \text {light }}$ ), oxidation and sample kept in darkness (pretO $\left.{ }_{2 \_ \text {dark }}\right)$ and reduction and sample exposed to light $\left(\mathrm{pretH}_{2}\right)$. The catalytic results are shown in Figure 1 and they are compared with those obtained by the thermal oxidation. As it can be seen for the samples pretreated with oxygen, depending on the presence of light, the catalytic behavior of the samples changes, obtaining the worst activity with the sample kept in darkness. On the other hand, the best activity was obtained with the sample pretreated with hydrogen or with the sample pretreated with oxygen and exposed to light. This result is suggesting that even distinct pretreatment conditions were applied, similar silver species are present in the reduced catalysts and in the oxidized one but exposed to light.

In order to determine the nature of these species, the samples were characterized by UV-Vis DRS as previous studies have demonstrated the possibility to discriminate different type of silver species by this technique [27-30]. Intense bands between 220 and $238 \mathrm{~nm}$ correspond to $\mathrm{Ag}^{+}$species, whereas $\mathrm{Ag}^{0}$ species are related to the bands around 320 and in the range 400$600 \mathrm{~nm}[21,31-33]$. The spectra are shown in Figure 2, observing a pronounced band around $490 \mathrm{~nm}$ in the sample pretreated with hydrogen $\left(\mathrm{Ag}_{\mathrm{imp}}\right.$ - pretH $\left._{2}\right)$, denoting the metallic state of the silver species in this sample. The same material after an oxidative treatment ( $\mathrm{Ag}_{\text {imp }}{ }^{-}$ pretO $\mathrm{O}_{2}$ ) and light exposed also displays a band around 420 and $322 \mathrm{~nm}$ confirming the presence of metallic silver species in this sample. Nevertheless, some $\mathrm{Ag}^{+}$species can be also present as it is suggested by the band at $225 \mathrm{~nm}$. Therefore, it can be concluded that cationic silver species present in the sample prepared by wetness impregnation are reduced to metallic silver during light exposure. This phenomenon has been previously described and has been explained by the auto-reduction of silver oxides produced by light [34]. In fact, as it is shown in Figure 1, if this sample was pretreated under an oxidative atmosphere but kept in darkness, it does not show significant activity, indicating that the active metallic silver species were not formed and silver remains as $\operatorname{Ag}(\mathrm{I})$. 


\subsection{Preparation method effect}

Another variable that can produce different silver species is the method used to incorporate silver into the zeolites. Figure 3 shows the results obtained with the Ag-catalysts containing the same silver content, but prepared by different methods, i.e. incipient wetness impregnation, ion exchange, and as silver-nanoclusters. The samples used for the comparison were pretreated with oxygen and exposed to light, as this was the simplest activation procedure. For a better comparison, the results obtained with the non-catalytic reaction and with the sample activated with oxygen and kept in darkness are also shown. As it can be seen the sample prepared with the impregnation method (light exposed) is the most active one, whilst the samples prepared by ion exchange or with Ag-nanoclusters have lower activity than the former one but higher than the catalyst pre-treated with oxygen and kept in darkness. These results show that most of the species present in the samples prepared by ion exchange or with the silver nanoclusters are different from those formed in the catalysts prepared by wetness impregnation. In order to identify the silver species, the samples were analyzed by UV-Vis spectroscopy. The spectra also appear in Figure 2 and mainly bands below $300 \mathrm{~nm}$ are observed in the silver ion-exchanged sample $\left.\left(\mathrm{Ag}_{\text {ex }}-\text { pretO}\right)_{2}\right)$, and in the ITQ2 with the nanoclusters $\left(\mathrm{Ag}_{\mathrm{x}}-\mathrm{pretO}_{2}\right)$. These bands have been assigned to silver cationic species [21, 3133], nevertheless the presence of some metallic silver in these materials cannot be excluded. In fact the catalytic results are between those obtained with the samples prepared by wetness impregnation kept in darkness (cationic silver) and those obtained with the same catalyst exposed to light (metallic silver). This suggests that a combination of both silver species $\left(\mathrm{Ag}^{0}\right.$ and $\mathrm{Ag}^{+}$) are present in the catalysts prepared by ion exchange and with the supported Agnanoclusters, indicating that cationic silver is more stabilized in these catalysts than in those prepared by wetness impregnation.

\subsection{Evolution of silver species in the Ag-nanoclusters}

The presence and evolution of the silver species under different thermal and oxidative/reduction treatments was studied with the catalysts prepared with silver nanoclusters supported on ITQ-2 zeolite. Those catalysts were used in successive reactions with intermediate activations (see Table 1) and the results obtained are shown in Figure 4. As it is observed, after a first reduction at $150^{\circ} \mathrm{C}$, the catalytic activity was similar to that obtained with the catalyst prepared by wetness impregnation and kept in darkness, where the silver was present as cationic species. The UV-Vis spectrum of this sample, shown in Figure 
$2\left(\mathrm{Ag}_{\mathrm{x}}\right.$ pret $\left.\mathrm{H}_{2}\right)$, present the main bands below to $300 \mathrm{~nm}$, corresponding to cationic silver species [21, 31-33], confirming the formation of cationic silver nanoclusters and indicating that metallic silver was not formed after this reductive treatment at $150^{\circ} \mathrm{C}$. After that, several consecutives treatments and reactions made up to $600^{\circ} \mathrm{C}$ (Table 1) have been done to the same Ag-nanoclusters supported on the ITQ-2 zeolite. As it can be observed, the activity of this sample increases while increasing the number of treatments and reactions. This suggests that partially charged cationic nanoclusters evolves during these thermal treatments to the formation of metallic nanoparticles as similar results to those obtained with the sample prepared by wetness impregnation and reduced with $\mathrm{H}_{2}$ were obtained. These results also reflect the stability of $\mathrm{Ag}^{\delta+}$ species in nanoclusters against reduction if compared with those present in the samples prepared by wetness impregnation. The last are immediately reduced to $\mathrm{Ag}^{0}$ by the presence of light, leading to a complete oxidation of $\mathrm{CO}$ at $400^{\circ} \mathrm{C}$ (Figure 2), whilst for a complete reduction of the silver species present in the nanoclusters, this is to obtain the same catalytic results, many runs at high temperatures with oxidative/reductive conditions are necessary.

The resolution of UV-Vis spectroscopy was not good enough to analyze these changes as many undefined bands with much noise were formed. Then the evolution of the $\mathrm{Ag}_{\mathrm{x}}$ nanoclusters supported on the ITQ2 was followed by XAS spectroscopy to get more insides from these differences. Figure 5 shows the XANES of the $\mathrm{Ag}_{\mathrm{x}}$ nanoclusters supported on the ITQ2 at Ag K-edge, fresh and after reaction. The XANES of Ag foil and AgO references, the initial supported $\mathrm{Ag}_{\mathrm{x}}$ nanocluster (fresh) catalysts as well as the catalyst after being pretreated under $\mathrm{H}_{2}$ and $\mathrm{O}_{2}$ atmosphere are shown in Figure S4. Compared to the $\mathrm{Ag}$ foil, the fresh sample shows slightly higher Ag K-edge white line intensity and broadening of the first peak above the edge, which is in agreement with the reported XANES of Ag nanocluster [6, 35]. The peak around $25.550 \mathrm{keV}$ is absent in the fresh supported $\operatorname{Ag}_{\mathrm{x}}$ nanoclusters, appearing only under oxidative pre-treatment and after reaction. Several groups have been previously observed that $\mathrm{Au}$ and $\mathrm{Ag}$ nanoclusters did not present this type of multiple scattering peaks like the foil, relating this with their small size [6, 36-38]. Therefore, it represents a fingerprint for the metal nanoclusters. In the compounds containing cationic silver, such as $\mathrm{Ag}_{2} \mathrm{~S}$ or $\mathrm{Ag}$ thiol, the Ag K-edge XANES spectra show obvious shifts in the positions and intensities of the white line and other peaks above the edge in comparison to Ag foil [35]. For Ag atoms forming covalent bond with $S$ the white line systematically shifts to lower energies. No energy edge shift was observed between the fresh sample and the sample after pretreatment 
under $\mathrm{H}_{2}$ atmosphere, indicating no alteration in the cluster structure and keeping the partially charge oxidation state. However, the sample after pretreatment under $\mathrm{O}_{2}$ atmosphere show a shift to higher energy and a decrease in the intensity of the white line, which could be related to the removal of the thiolate ligands and to an increase of the metallic oxidation state portion inside the cluster. The stability of the cluster and changes in oxidation state of the supported $\mathrm{Ag}$ nanoclusters, after $\mathrm{CO}$ oxidation reaction at different temperatures, can be observed in Figure 5. The sample pre-treated under $\mathrm{H}_{2}$ shows a spectrum slightly shifted to higher energy together with more defined features around $25550 \mathrm{eV}$, more similar to $\mathrm{Ag}$ foil. It could be related to the removal of thiolate ligands during the reaction, increasing the metallic oxidation state of the cluster.

Therefore, the preliminary XANES analysis confirms the previous hypothesis and they are in agreement with the previous DRS studies. As it can be observed in Figure 5 the fresh sample evolves towards the formation of metallic silver during the $\mathrm{CO}$ oxidation reaction mainly if the sample has been activated at high temperatures. This can explain the increase of the activity observed in the successive cycles, obtaining after the third run the same results than those obtained with the catalyst prepared by wetness impregnation and reduced in hydrogen, were mainly metallic silver is formed.

\section{CONCLUSIONS}

The results obtained show that the three preparation methods used for the incorporation of silver to the zeolite and the different pretreatments of the samples lead to different silver species configuration on the surface of the ITQ2. These species can be identified with the CO oxidation reaction as they have different catalytic behavior. The active phases for the $\mathrm{CO}$ oxidation are the metallic silver sites, whilst cationic silver is not active in this reaction. Metallic silver is formed on the samples prepared by wetness impregnation due to the silver auto-reduction by light whilst cationic silver is formed on the samples prepared by ion exchange and in the silver nanoclusters. The partially charged silver species formed on this sample are very stable as high temperatures and presence of hydrogen are required for the formation of metallic silver, this is to be active for the $\mathrm{CO}$ oxidation reaction. Therefore, it can be concluded that the $\mathrm{CO}$ oxidation reaction can be used as a tool to disclose different silver species in the supported Ag catalysts. 


\section{Acknowledgements}

The authors thank the Spanish Ministry of Economy and Competitiveness through RTI2018-101784-B-I00 (MINECO/FEDER) and SEV-2016-0683 projects for the financial support. We gratefully acknowledge ALBA synchrotron for allocating beamtime (proposal 2015091414) and the CLÆSS beamline staff for their help and technical support during our experiment. CG and NB thank the TUW Innovative Project GIP165CDGC. CG, SP, VT, NB and GR are thankful for financial support from the Austrian Science Fund (FWF) through projects DK+ Solids4Fun (W1243) and ComCat (I 1041-N28). I. López Hernández is grateful to Generalitat Valenciana and European Social Fund for the pre doctoral grant ACIF2017. 


\section{REFERENCES}

[1] N. Serhan, A. Tsolakis, A. Wahbi, F.J. Martos, S. Golunski, Modifying catalytically the soot morphology and nanostructure in diesel exhaust: Influence of silver De-NOx catalyst (Ag/A12O3), Applied Catalysis B: Environmental, 241 (2019) 471-482.

[2] K. Góra-Marek, K.A. Tarach, Z. Piwowarska, M. Łaniecki, L. Chmielarz, Ag-loaded zeolites Y and USY as catalysts for selective ammonia oxidation, Catalysis Science \& Technology, 6 (2016) 1651-1660.

[3] X. Hu, J. Bai, H. Hong, C. Li, Supercritical carbon dioxide anchored highly dispersed silver nanoparticles on 4A-zeolite and selective oxidation of styrene performance, CrystEngComm, 18 (2016) 2469-2476.

[4] J.L. Cerrillo, A.E. Palomares, F. Rey, S. Valencia, M.B. Pérez-Gago, D. Villamón, L. Palou, Functional Ag-Exchanged Zeolites as Biocide Agents, ChemistrySelect, 3 (2018) 4676-4682.

[5] X.-Y. Dong, Z.-W. Gao, K.-F. Yang, W.-Q. Zhang, L.-W. Xu, Nanosilver as a new generation of silver catalysts in organic transformations for efficient synthesis of fine chemicals, Catalysis Science \& Technology, 5 (2015) 2554-2574.

[6] K.O. Sulaiman, V. Sudheeshkumar, R.W.J. Scott, Activation of atomically precise silver clusters on carbon supports for styrene oxidation reactions, RSC Advances, 9 (2019) 2801928027.

[7] E. Coutino-Gonzalez, W. Baekelant, J.A. Steele, C.W. Kim, M.B.J. Roeffaers, J. Hofkens, Silver Clusters in Zeolites: From Self-Assembly to Ground-Breaking Luminescent Properties, Acc Chem Res, 50 (2017) 2353-2361.

[8] L. Liu, A. Corma, Metal Catalysts for Heterogeneous Catalysis: From Single Atoms to Nanoclusters and Nanoparticles, Chemical Reviews, 118 (2018) 4981-5079.

[9] J. Zhao, R. Jin, Heterogeneous catalysis by gold and gold-based bimetal nanoclusters, Nano Today, 18 (2018) 86-102.

[10] C. García, S. Pollitt, M. van der Linden, V. Truttmann, C. Rameshan, R. Rameshan, E. Pittenauer, G. Allmaier, P. Kregsamer, M. Stöger-Pollach, N. Barrabés, G. Rupprechter, Support effect on the reactivity and stability of Au25(SR)18 and Au144(SR)60 nanoclusters in liquid phase cyclohexane oxidation, Catalysis Today, (2018).

[11] B. Zhang, S. Kaziz, H. Li, M.G. Hevia, D. Wodka, C. Mazet, T. Burgi, N. Barrabes, Modulation of Active Sites in Supported Au-38(SC2H4Ph)(24) Cluster Catalysts: Effect of Atmosphere and Support Material, J Phys Chem C, 119 (2015) 11193-11199. 
[12] B. Zhang, A. Sels, G. Salassa, S. Pollitt, V. Truttmann, C. Rameshan, J. Llorca, W.

Olszewski, G. Rupprechter, T. Burgi, N. Barrabes, Ligand Migration from Cluster to Support: A Crucial Factor for Catalysis by Thiolate-protected Gold Clusters, Chemcatchem, 10 (2018) 5372-5376.

[13] Frontiers of Nanoscience, in: T. Tsukuda, H. Häkkinen (Eds.) Frontiers of Nanoscience, Elsevier2015, pp. ii.

[14] G. Natarajan, A. Mathew, Y. Negishi, R.L. Whetten, T. Pradeep, A Unified Framework for Understanding the Structure and Modifications of Atomically Precise Monolayer Protected Gold Clusters, Journal of Physical Chemistry C, 119 (2015) 27768-27785. [15] T. Tsukuda, H. Häkkinen, Chapter 1 - Introduction, in: T. Tsukuda, H. Häkkinen (Eds.) Frontiers of Nanoscience, Elsevier2015, pp. 1-7.

[16] X. Zhang, Z. Qu, X. Li, M. Wen, X. Quan, D. Ma, J. Wu, Studies of silver species for low-temperature $\mathrm{CO}$ oxidation on $\mathrm{Ag} / \mathrm{SiO} 2$ catalysts, Separation and Purification Technology, 72 (2010) 395-400.

[17] E. Kolobova, A. Pestryakov, G. Mamontov, Y. Kotolevich, N. Bogdanchikova, M. Farias, A. Vosmerikov, L. Vosmerikova, V. Cortes Corberan, Low-temperature CO oxidation on Ag/ZSM-5 catalysts: Influence of Si/Al ratio and redox pretreatments on formation of silver active sites, Fuel, 188 (2017) 121-131.

[18] A. Ausavasukhi, S. Suwannaran, J. Limtrakul, T. Sooknoi, Reversible interconversion behavior of Ag species in AgHZSM-5: XRD, 1H MAS NMR, TPR, TPHE, and catalytic studies, Applied Catalysis A: General, 345 (2008) 89-96.

[19] C. Shi, M. Cheng, Z. Qu, X. Bao, On the correlation between microstructural changes of Ag-H-ZSM-5 catalysts and their catalytic performances in the selective catalytic reduction of NOx by methane, Journal of Molecular Catalysis A: Chemical, 235 (2005) 35-43.

[20] D.S. Afanasev, O.A. Yakovina, N.I. Kuznetsova, A.S. Lisitsyn, High activity in CO oxidation of Ag nanoparticles supported on fumed silica, Catalysis Communications, 22 (2012) 43-47.

[21] E. Kolobova, A. Pestryakov, A. Shemeryankina, Y. Kotolevich, O. Martynyuk, H.J. Tiznado Vazquez, N. Bogdanchikova, Formation of silver active states in Ag/ZSM-5 catalysts for CO oxidation, Fuel, 138 (2014) 65-71.

[22] S. Royer, D. Duprez, Catalytic Oxidation of Carbon Monoxide over Transition Metal Oxides, Chemcatchem, 3 (2011) 24-65.

[23] N.K. Soliman, Factors affecting CO oxidation reaction over nanosized materials: A review, Journal of Materials Research and Technology, 8 (2019) 2395-2407. 
[24] M. Du, D. Sun, H. Yang, J. Huang, X. Jing, T. Odoom-Wubah, H. Wang, L. Jia, Q. Li, Influence of $\mathrm{Au}$ Particle Size on $\mathrm{Au} / \mathrm{TiO} 2$ Catalysts for CO Oxidation, The Journal of Physical Chemistry C, 118 (2014) 19150-19157.

[25] V.Fornés, A. Corma, J.M. Guil, S. Pergher, Th.L.M. Maesen, J.G. Buglass, Preparation, characterisation and catalytic activity of ITQ-2, a delaminated zeolite, Microporous and Mesoporous Materials, 38 (2000) 301-309.

[26] C.P. Joshi, M.S. Bootharaju, M.J. Alhilaly, O.M. Bakr, [Ag25(SR)18](-): The "Golden" Silver Nanoparticle, J Am Chem Soc, 137 (2015) 11578-11581.

[27] S.G. Aspromonte, M.D. Mizrahi, F.A. Schneeberger, J.M.R. López, A.V. Boix, Study of the Nature and Location of Silver in Ag-Exchanged Mordenite Catalysts. Characterization by Spectroscopic Techniques, The Journal of Physical Chemistry C, 117 (2013) 25433-25442. [28] G. Veronesi, A. Deniaud, T. Gallon, P.H. Jouneau, J. Villanova, P. Delangle, M.

Carriere, I. Kieffer, P. Charbonnier, E. Mintz, I. Michaud-Soret, Visualization, quantification and coordination of $\mathrm{Ag}+$ ions released from silver nanoparticles in hepatocytes, Nanoscale, 8 (2016) 17012-17021.

[29] G. Veronesi, C. Aude-Garcia, I. Kieffer, T. Gallon, P. Delangle, N. Herlin-Boime, T. Rabilloud, M. Carriere, Exposure-dependent Ag+ release from silver nanoparticles and its complexation in AgS2 sites in primary murine macrophages, Nanoscale, 7 (2015) 7323-7330. [30] N.V. Hudson-Smith, P.L. Clement, R.P. Brown, M.O.P. Krause, J.A. Pedersen, C.L. Haynes, Research highlights: speciation and transformations of silver released from Ag NPs in three species, Environmental Science-Nano, 3 (2016) 1236-1240.

[31] K.-i. Shimizu, K. Sugino, K. Kato, S. Yokota, K. Okumura, A. Satsuma, Formation and Redispersion of Silver Clusters in Ag-MFI Zeolite as Investigated by Time-Resolved QXAFS and UV-Vis, The Journal of Physical Chemistry C, 111 (2007) 1683-1688.

[32] D. Chen, Z. Qu, S. Shen, X. Li, Y. Shi, Y. Wang, Q. Fu, J. Wu, Comparative studies of silver based catalysts supported on different supports for the oxidation of formaldehyde, Catalysis Today, 175 (2011) 338-345.

[33] F. Schuricht, W. Reschetilowski, Simultaneous selective catalytic reduction (SCR) of NOx and N2O over Ag/ZSM-5 - Catalytic studies and mechanistic implications, Microporous and Mesoporous Materials, 164 (2012) 135-144.

[34] S.K.B. Deepak B. Akolekar, Adsorption ofNO and CO on silver-exchanged microporous materials, Journal of Molecular Catalysis A: Chemical, 157 (2000) 199-206.

[35] J. Liu, K.S. Krishna, C. Kumara, S. Chattopadhyay, T. Shibata, A. Dass, C.S.S.R. Kumar, Understanding Au similar to $98 \mathrm{Ag}$ similar to 46(SR)(60) nanoclusters through 
investigation of their electronic and local structure by X-ray absorption fine structure, RSC Adv., 6 (2016) 25368-25374.

[36] D.M. Chevrier, R. Yang, A. Chatt, P. Zhang, Bonding properties of thiolate-protected gold nanoclusters and structural analogs from X-ray absorption spectroscopy, Nanotechnol Rev, 4 (2015) 193-206.

[37] S. Yamazoe, T. Tsukuda, X-ray Absorption Spectroscopy on Atomically Precise Metal Clusters, Bulletin of the Chemical Society of Japan, 92 (2019) 193-204.

[38] B. Zhang, A. Sels, G. Salassa, S. Pollitt, V. Truttmann, C. Rameshan, J. Llorca, W. Olszewski, G. Rupprechter, T. Bürgi, N. Barrabés, Ligand Migration from Cluster to Support: A Crucial Factor for Catalysis by Thiolate-protected Gold Clusters, ChemCatChem, 10 (2018) 5372-5376. 


\section{CAPTION TO FIGURES}

Figure 1. Influence of the catalyst pre-treatment in the $\mathrm{CO}$ oxidation reaction (Ag-ITQ2 catalyst prepared by wetness impregnation). (匚) non catalyzed reaction; $\left({ }^{\circ}\right) \mathrm{O}_{2}$ pre-treatment and darkness; $\left(\mathrm{O}_{2}\right.$ pre-treatment and light exposed; $\left(\mathrm{O}_{2} \mathrm{H}_{2}\right.$ pre-treatment.

Figure 2. UV-Vis DRS spectra of the Ag-ITQ2 samples prepared by different methods (ex: silver ion exchanged; imp: silver wetness impregnation; $x$ : silver nanoclusters) and with different pretreatments (reduction: $\operatorname{pret}_{2}$; oxidation: $\operatorname{pretO}_{2}$ ).

Figure 3. Influence of the method used for the catalyst silver addition (samples pretreated

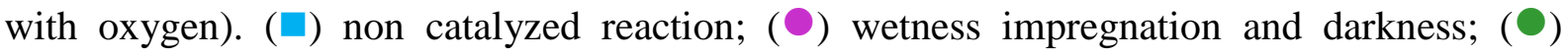
wetness impregnation and light exposed; $(\diamond)$ ion exchanged light exposed; $(\Delta)$ silver nanoclusters, light exposed.

Figure 4. Activity of the silver nanoclusters supported on the ITQ-2 in successive reactions. $(\square)$ non catalyzed reaction, $(\Delta)$ run $1,(\square)$ run 2, ( $\square)$ run 3, ( $)$ run 4, ( $)$ wetness impregnation with $\mathrm{H}_{2}$ pre-treatment.

Figure 5. Ag K-edge XANES spectra of references and of Ag nanoclusters supported on ITQ2 fresh and after $\mathrm{CO}$ oxidation reaction till $400^{\circ} \mathrm{C}$, with different pretreatments. 


\title{
Supporting information
}

\section{EVALUATION OF THE SILVER SPECIES NATURE IN Ag-ITQ2 ZEOLITES BY THE CO OXIDATION REACTION}

\author{
I. López-Hernández 1, C. García ${ }^{2}$, V. Truttmann ${ }^{2}$, S. Pollitt ${ }^{2}$, N. Barrabés ${ }^{2}$, G. \\ Rupprechter $^{2}$, F. Rey ${ }^{1}$, A. E. Palomares ${ }^{1}$
}

${ }^{1}$ Instituto de Tecnología Química, Universitat Politècnica de València - Consejo Superior de Investigaciones Científicas (UPV-CSIC), Valencia, España

${ }^{2}$ Institute of Materials Chemistry, Technical University Vienna, Viena, Austria

Corresponding author: apalomar@iqn.upv.es

The purity of the synthesized $\mathrm{Ag}_{25}\left(\mathrm{SPhMe}_{3}\right)_{18} \mathrm{PPh}_{4}$ cluster solution was confirmed by UV-Vis (Figure S1-left) [1]. The absorption spectra of Ag nanoclusters exhibit characteristic features caused by electronic transitions between different molecular orbitals, being directly related to the cluster structure. In this way the characteristic band at $490 \mathrm{~nm}$ is observed together with a wide band with a maximum at ca. $700 \mathrm{~nm}$. Figure S1 also shows the FTIR spectra that confirms the bonding between $\mathrm{Ag}$ and $\mathrm{S}$ by the absence of the $\mathrm{S}-\mathrm{H}$ stretching vibration, expected around $2525 \mathrm{~cm}^{-1}$, whereas the characteristic $\mathrm{C}-\mathrm{H}, \mathrm{C}-\mathrm{S}$ and aromatic stretching bands of the thiolated ligand were indeed observed. In addition, the spectrum shows an unexpected strong band at $1000-1200 \mathrm{~cm}^{-1}$.
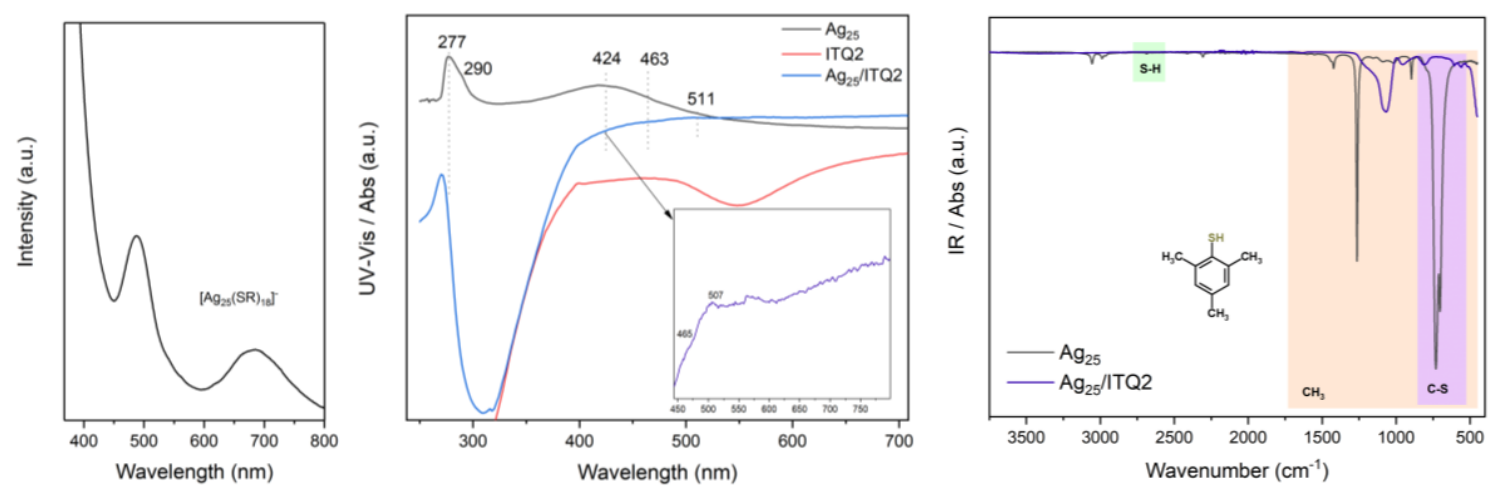

Fig S1. UV-Vis spectra of $A g_{25}$ nanoclusters in solution after synthesis (left); UV-Vis DRS spectra of $A g_{x}, I T Q 2$ and $A g_{x} / I T Q 2$ samples (middle); ATR-IR spectra of $A g_{25}$ nanoclusters in solution and upported, $A g_{x} / I T Q 2$ (right) 


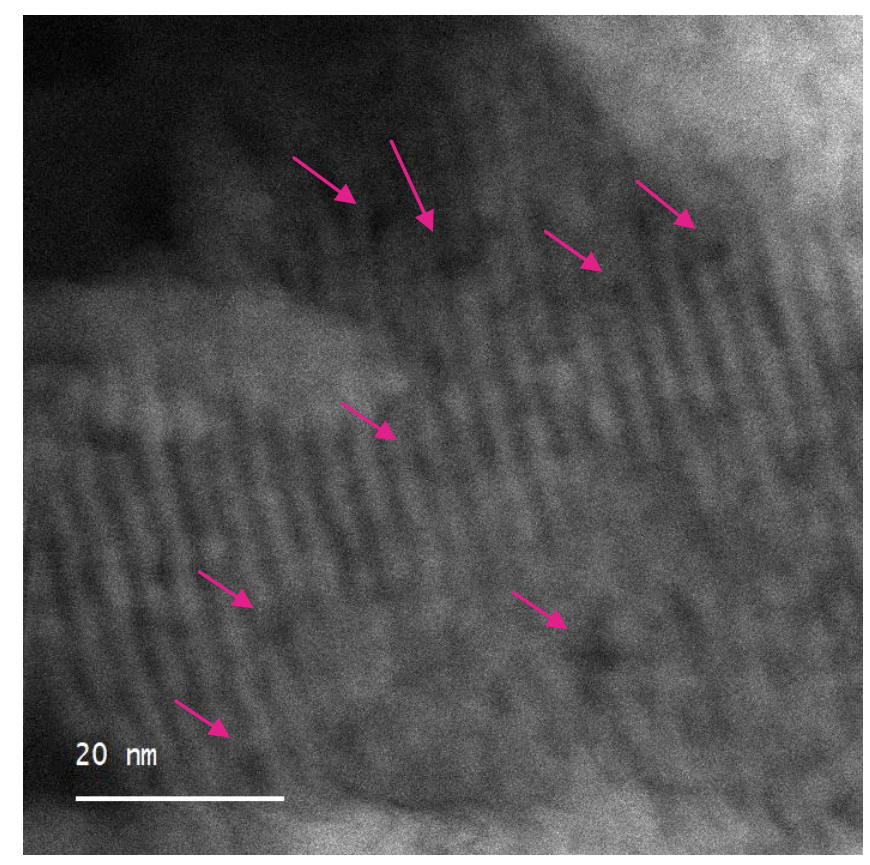

Fig S2. TEM image of $A g_{x}$ nanoclusters supported on ITQ2 zeolite.

The TEM image of the supported $\mathrm{Ag}_{\mathrm{x}}$ nanoclusters on the zeolite is displayed in Figure $\mathrm{S} 2$. Due to the small particle size, it is difficult to analyze the supported nanoclusters by this technique but the small dark dots observed can be related to $\operatorname{Ag}_{\mathrm{x}}$ nanoclusters.

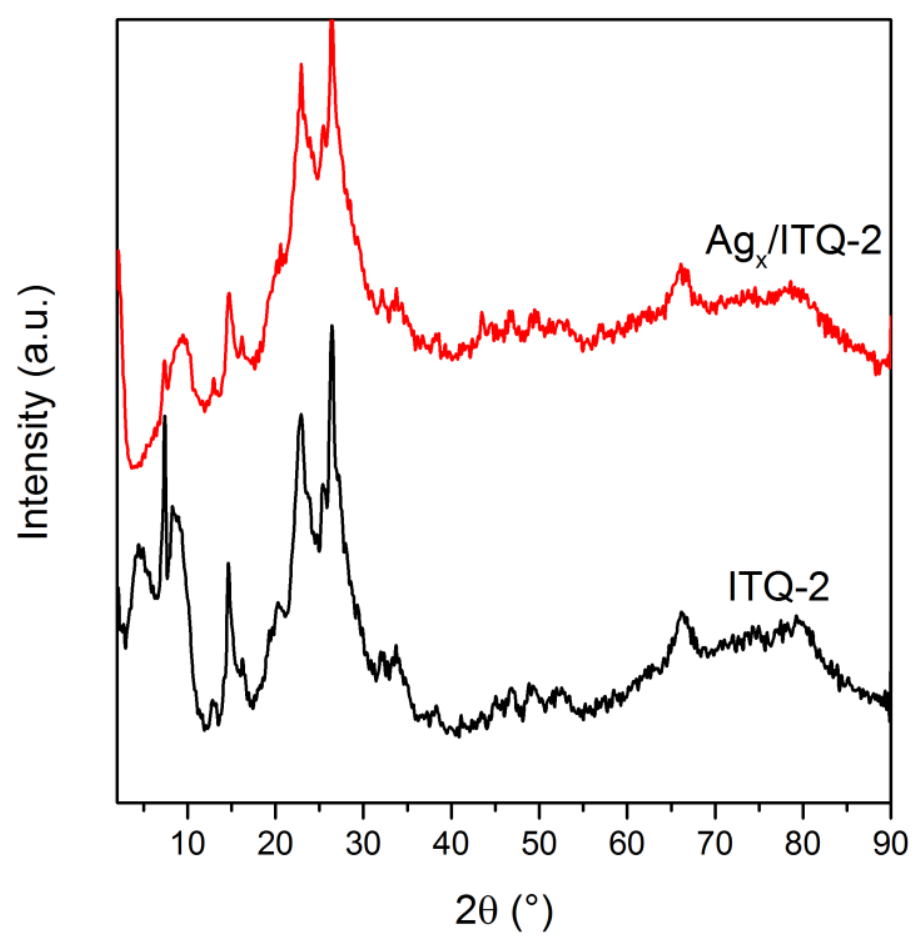

Fig S3. ITQ2-zeolite and $A g_{x} / I T Q X R D$ patterns 

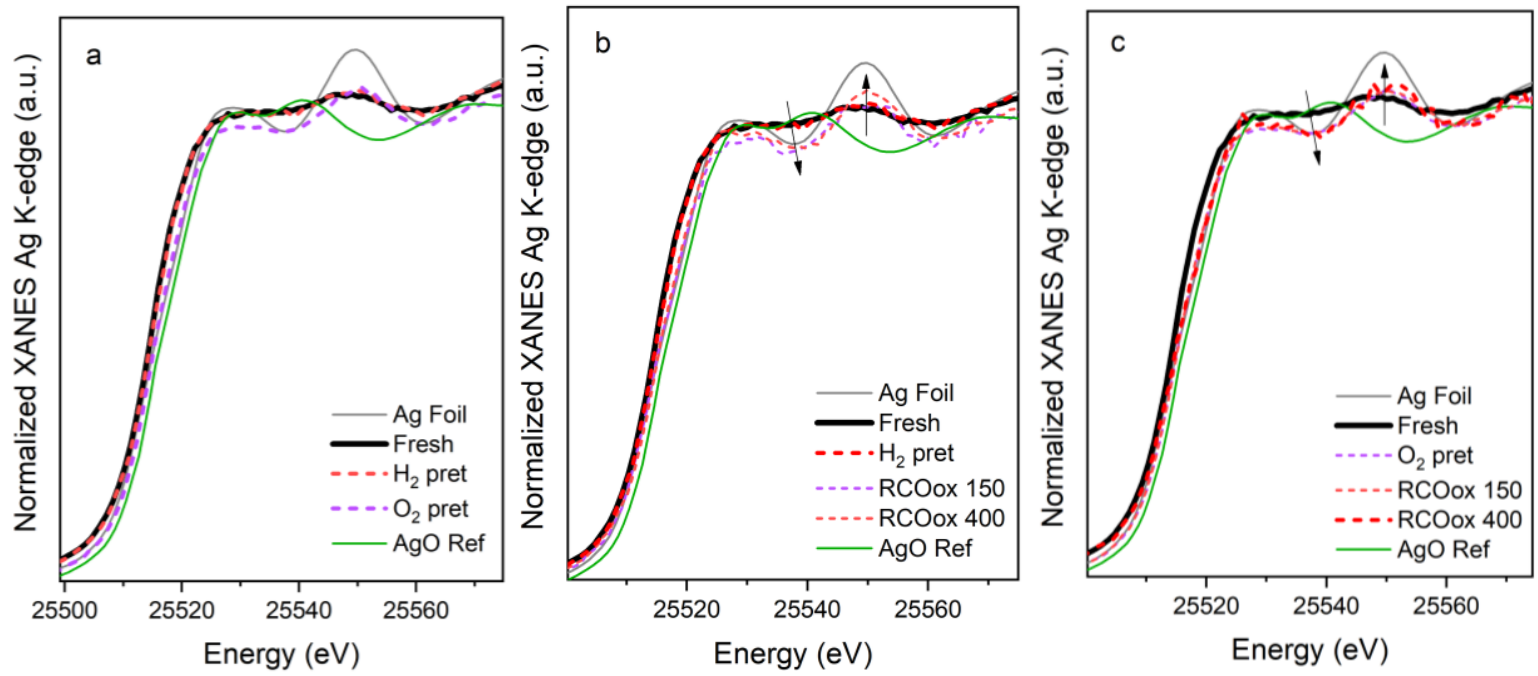

Fig S4. Ag K-edge XANES spectra of $A g_{x} / I T Q 2$ (fresh), after pretreatments under different atmospheres and after $\mathrm{CO}$ oxidation reaction. References of $\mathrm{Ag}$ in metallic and oxidation state are also included for comparison.

[1] C.P. Joshi, M.S. Bootharaju, M.J. Alhilaly, O.M. Bakr, [Ag25(SR)18]-: The "Golden" Silver Nanoparticle, Journal of the American Chemical Society, 137 (2015) 11578-11581. 
Table 1. Successive reactions made with the ITQ2 zeolite containing Ag-nanoclusters

\begin{tabular}{|c|l|}
\hline run & \multicolumn{1}{|c|}{ Treatment/Reaction } \\
\hline $\mathbf{1}$ & Activation with $\mathrm{H}_{2}-150^{\circ} \mathrm{C}$ and reaction $\left(\mathrm{CO}+\mathrm{O}_{2}\right.$ up to $\left.650^{\circ} \mathrm{C}\right)$ \\
\hline $\mathbf{2}$ & Same catalyst used in run 1 and reaction $\left(\mathrm{CO}+\mathrm{O}_{2}\right.$ up to $\left.600^{\circ} \mathrm{C}\right)$ \\
\hline $\mathbf{3}$ & $\begin{array}{l}\text { Same catalyst used in run 2, re activated with } \mathrm{H}_{2}-150^{\circ} \mathrm{C} \text { and reaction }\left(\mathrm{CO}+\mathrm{O}_{2} \text { up }\right. \\
\left.\text { to } 400^{\circ} \mathrm{C}\right)\end{array}$ \\
\hline $\mathbf{4}$ & $\begin{array}{l}\text { Same catalyst used in run 3, re activated with } \mathrm{H}_{2}-400^{\circ} \mathrm{C} \text { and reaction }\left(\mathrm{CO}+\mathrm{O}_{2} \text { up }\right. \\
\left.\text { to } 400^{\circ} \mathrm{C}\right)\end{array}$ \\
\hline
\end{tabular}




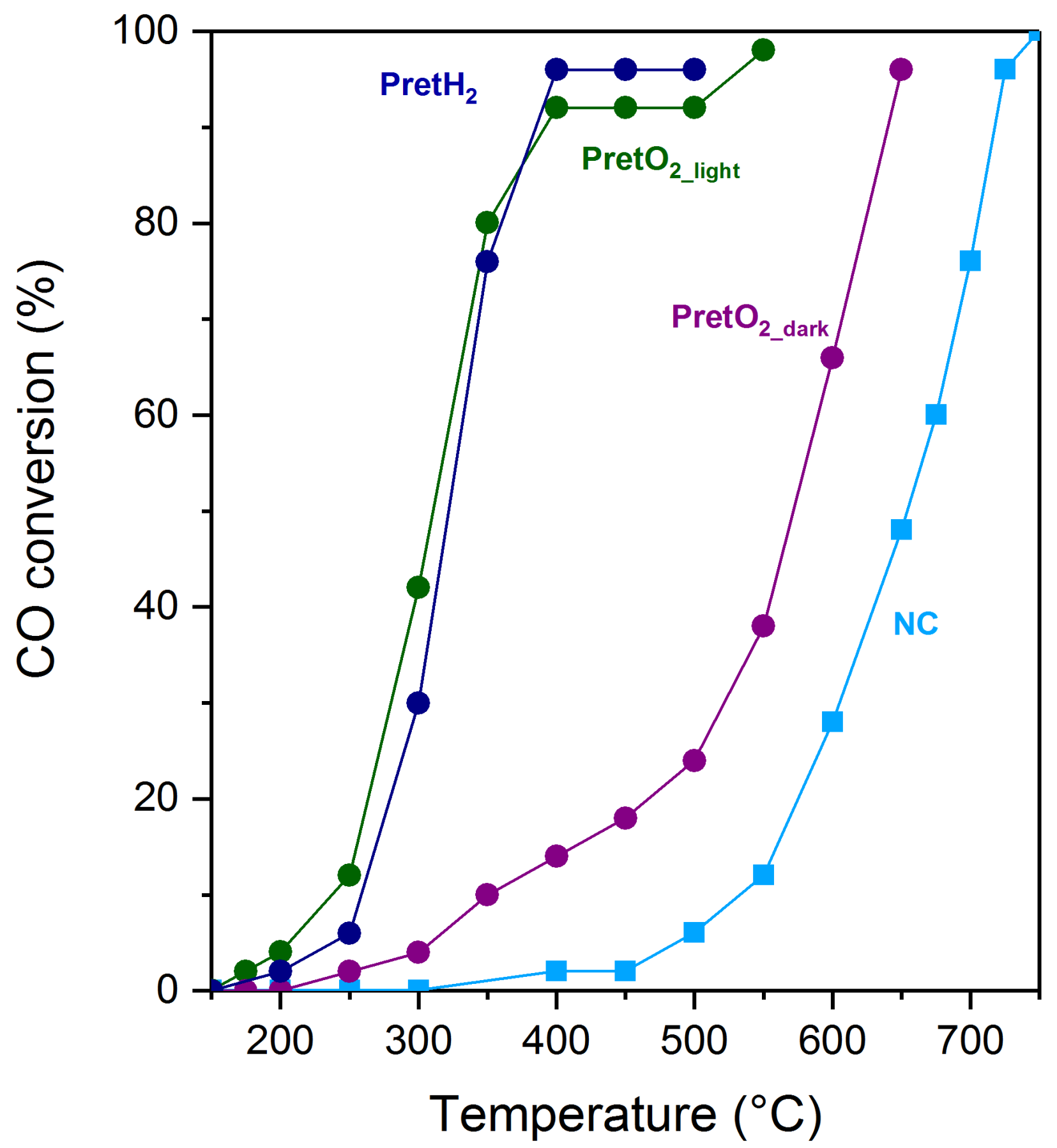




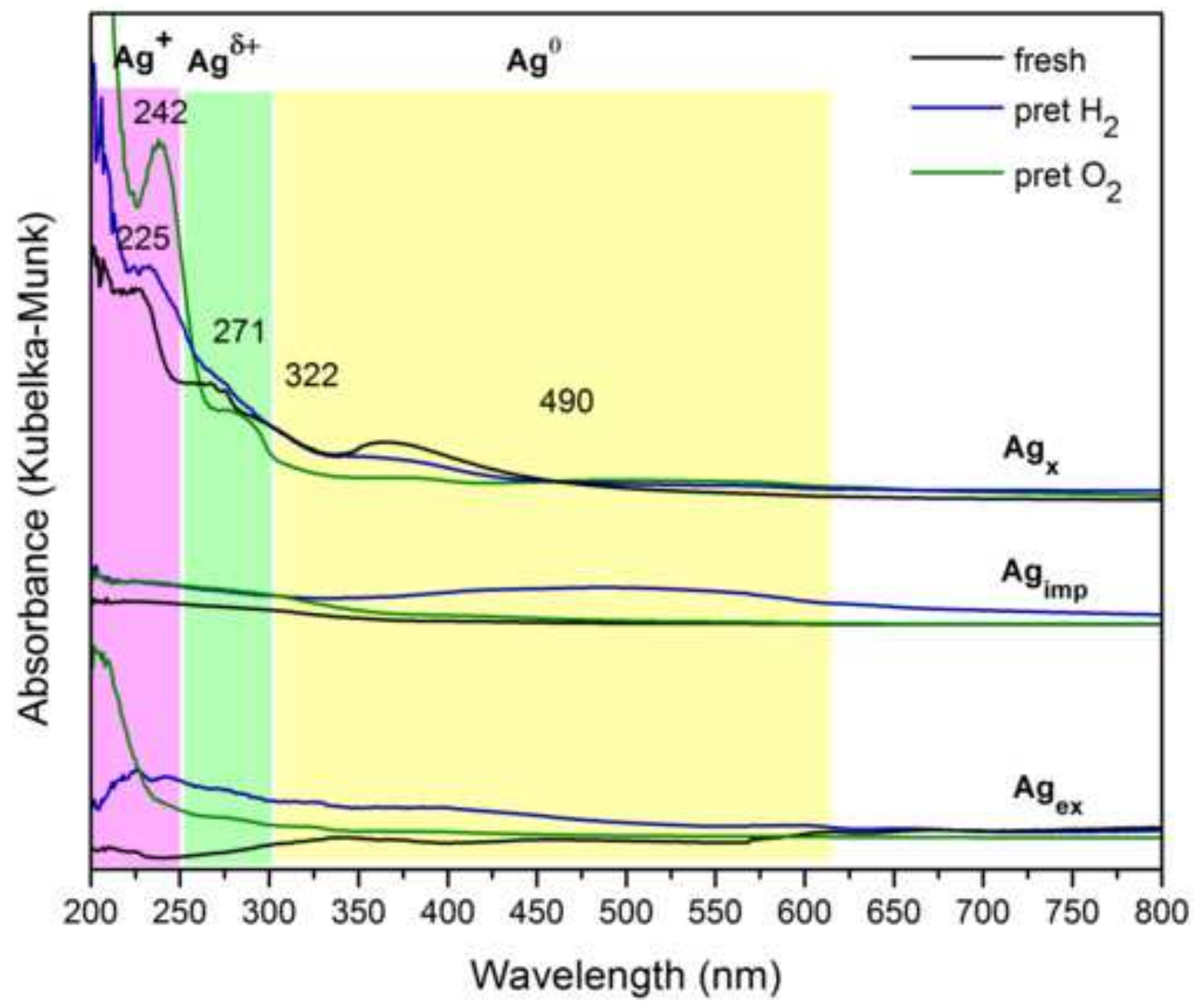




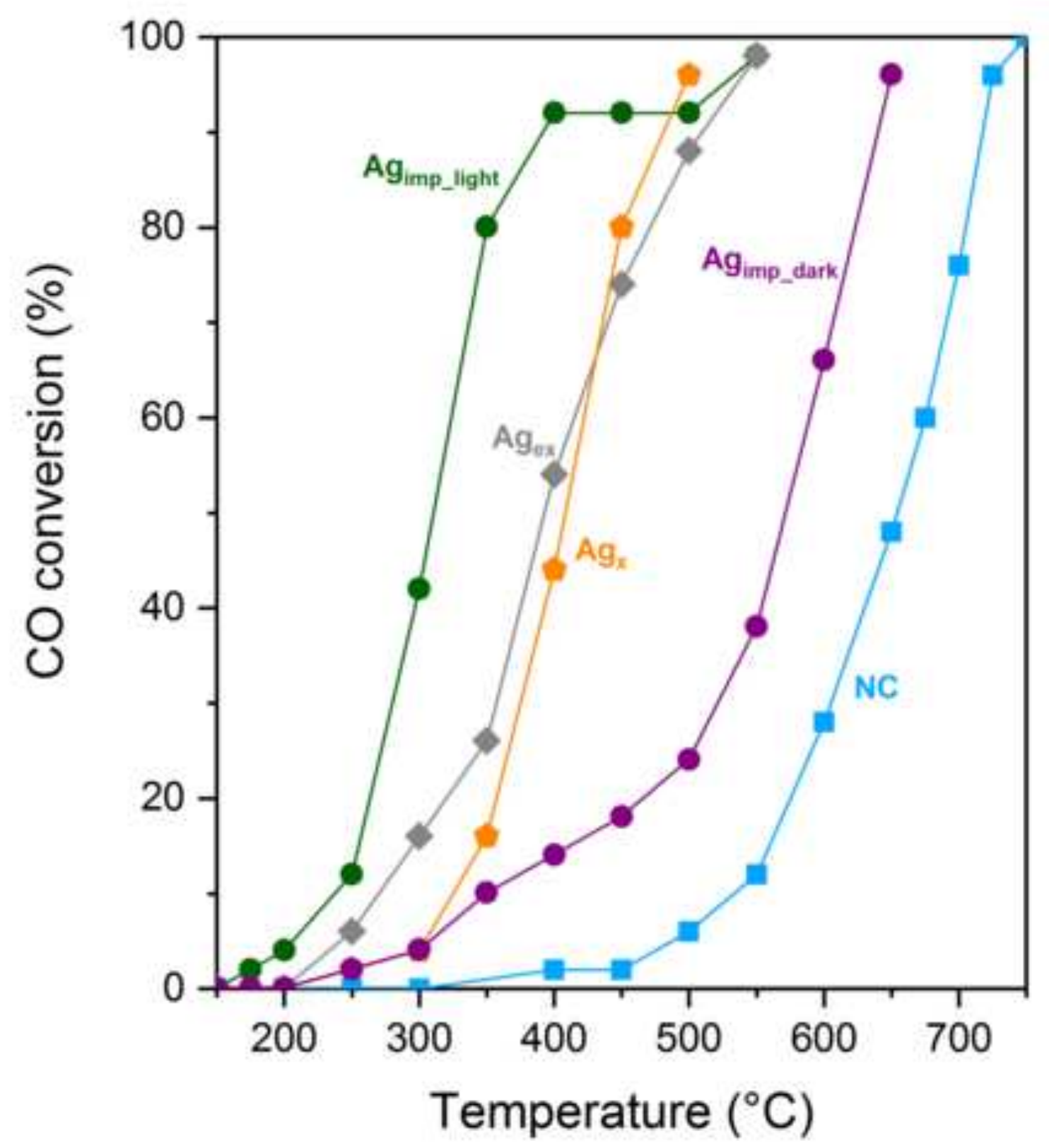


Click here to download high resolution image

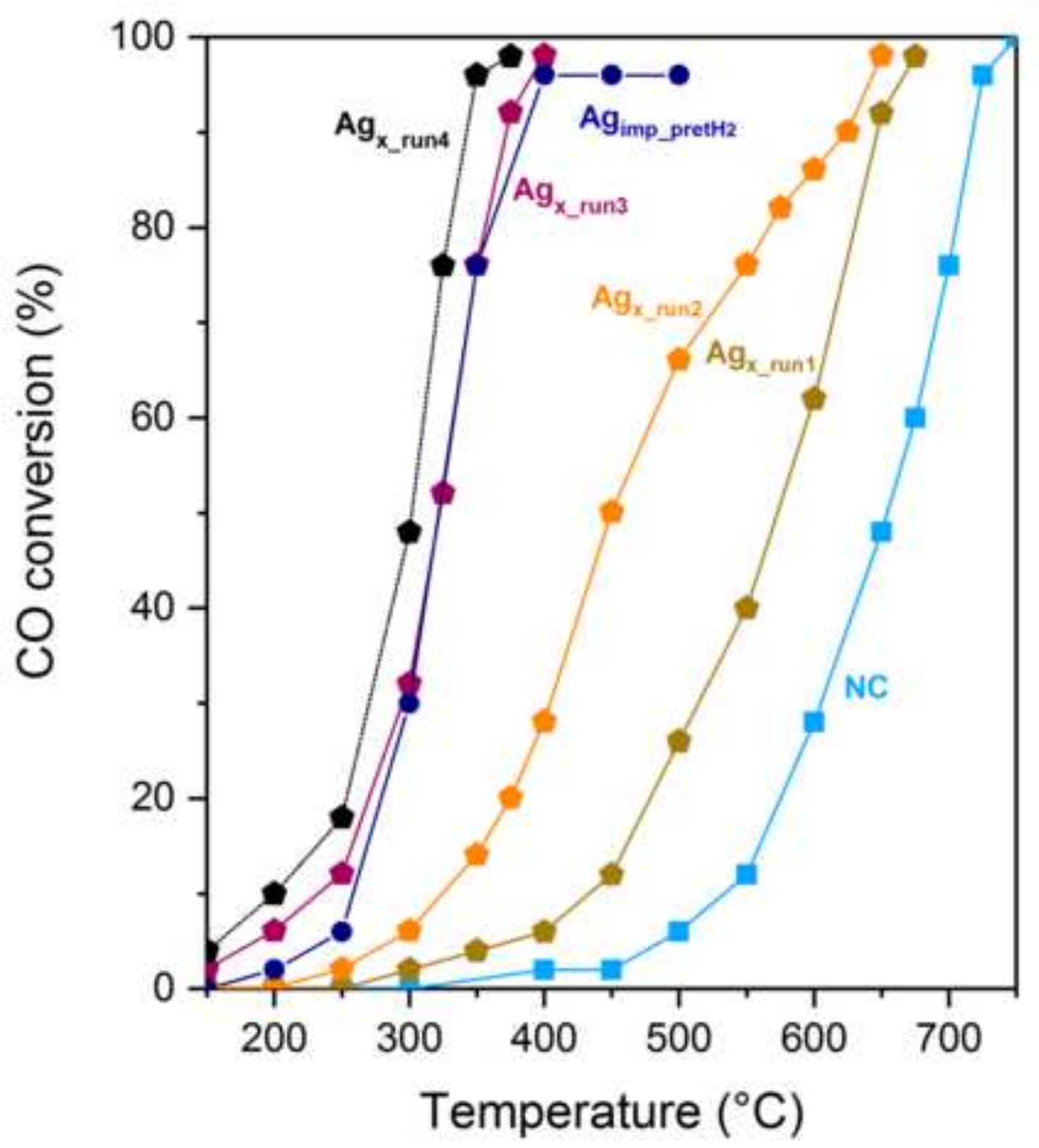




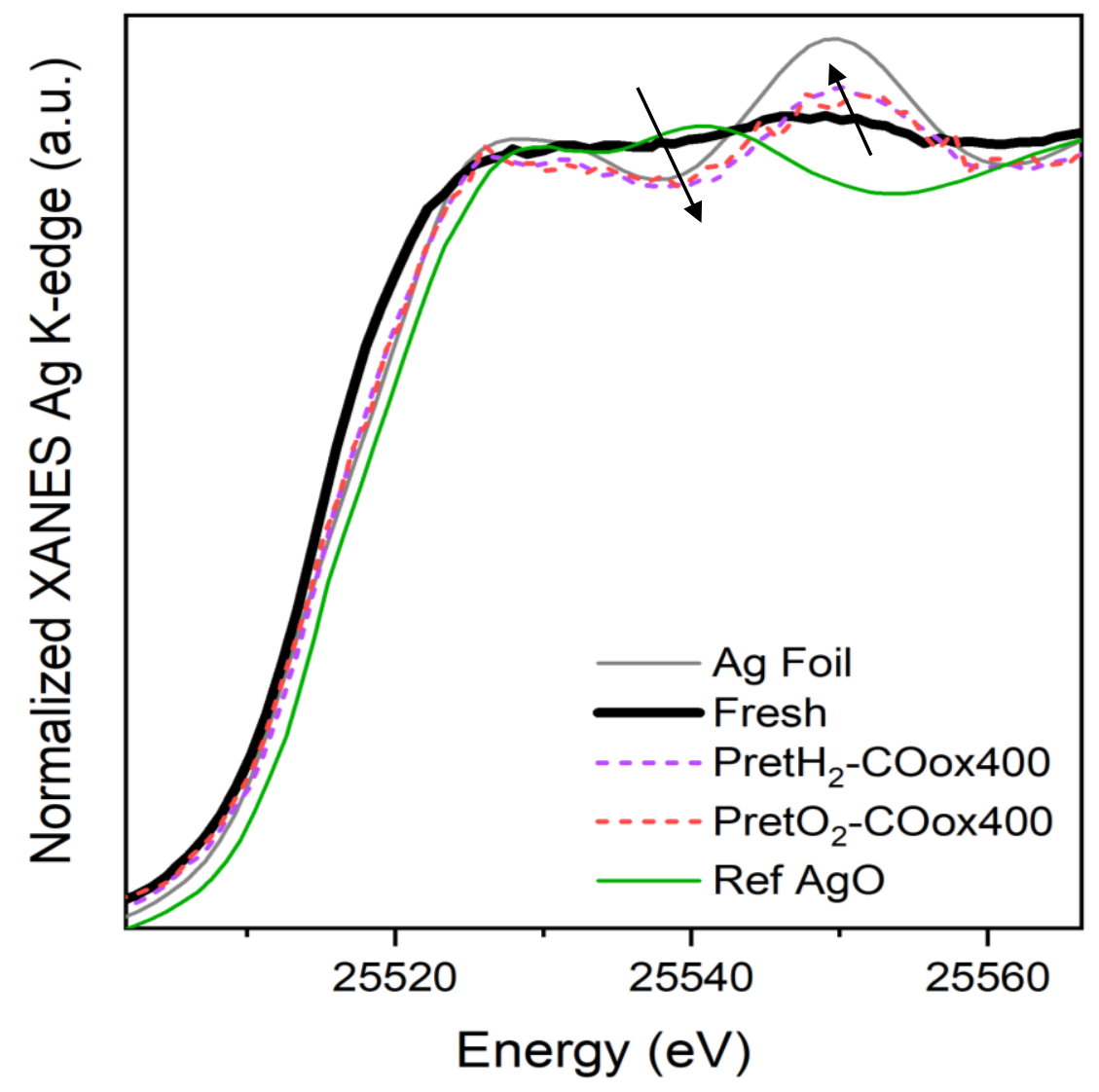

